\section{Mudanças recentes no financiamento federal do Sistema Único de Saúde: atenção básica à saúde}

\section{Recent changes of the Brazilian Public Health System federal financing: primary health}

Jorge José Santos Pereira Solla ${ }^{1}$

Ademar Arthur Chioro dos Reis ${ }^{2}$

Ana Paula Menezes Soter ${ }^{3}$

Afra Suassuna Fernandes 4

José João Lanceiro de Palma ${ }^{5}$

1 Centro Administrativo. Secretaria de Saúde da Bahia. Salvador, BA, Brasil.

2 Universidade Santa Cecília. Santos, SP, Brasil.

3,4 Instituto Materno Infantil Prof. Fernando Figueira-IMIP. Rua dos Coelhos, 300. Recife, PE, Brasil. CEP: 50.070-550

E-mail: ana.soter@imip.org.br

5 Ministério da Saúde. Brasília, DF, Brasil.

\begin{abstract}
The article presents a revision on the financing policy of the basic attention, along the prosecute of Public Health System (SUS) implementation, having the Family Health Strategy as propulsive element of the strong observed expansion in this attention component to the health. It argues the role of the Operational Rules of SUS, especially of the Basic Operational NormaNOB 01/96 and the Basic Attention Floor (PAB) while mark for investments enlargement in the basic attention and the importance of the constitutional amendment 29, while important legal instrument for investments increment of the three government's levels in the health. Given starting from supplied by national data banks, it analyzes the evolution of the financial values transferred fund thoroughly for the municipal districts, their populations' corrections and criteria incorporation for inequalities correction. It highlights the administration federal level strong inductor role of SUS, who by means of financing new mechanisms guaranteed coverage strong expansion of the basic attention, through the family health teams and professionals' incorporation of buccal health, in this assistance level.
\end{abstract}

Key words Decentralization, SUS (BR), Primary health care, Health financing

\section{Resumo}

$O$ artigo apresenta uma revisão sobre a política de financiamento da atenção básica, ao longo do processo de implementação do Sistema Único de Saúde (SUS), tendo a estratégia de saúde da família como elemento propulsor da forte expansão observada nesse componente da atenção à saúde. Discute o papel das Normas Operacionais do SUS, em especial da Norma Operacional Básica-NOB 01/96 e a implantação do Piso da Atenção Básica (PAB), enquanto marco para ampliação dos investimentos na atenção básica e a importância da emenda constitucional 29, enquanto instrumento legal relevante para incremento de investimentos dos três níveis de governo na saúde. A partir de dados fornecidos por bancos nacionais, analisa a evolução dos valores financeiros transferidos fundo a fundo para os municípios, as suas correções populacionais e incorporação de critérios para correção de desigualdades. Destaca o forte papel indutor do nível federal de gestão do SUS, que por meio de novos mecanismos de financiamento garantiu forte expansão de cobertura da atenção básica, por intermédio das equipes de saúde da família e incorporação dos profissionais de saúde bucal, neste nível de assistência.

Palavras-chave Descentralização, SUS (BR), Atenção básica, Financiamento da saúde 
A construção do Sistema Único de Saúde (SUS) na última década foi marcada por uma forte expansão da atenção básica, tendo na estratégia de Saúde da Família o seu principal propulsor. Este processo se deu com forte indução do governo federal e pequena participação dos governos estaduais, ocorrendo em paralelo, em um caminho de mútuo fortalecimento, a reorganização (na maioria dos municípios a implantação) da atenção básica e a ampliação das responsabilidades da gestão local do SUS. ${ }^{1}$

A participação relativa dos municípios no financiamento do SUS foi progressivamente aumentando, em especial no tocante à atenção básica. Enquanto em 1993 o governo federal contribuía com $72 \%$ dos recursos para o SUS e os municípios com $16 \%$ em 2002 estes percentuais tinham se alterado para $58 \%$ e $22 \%$, respectivamente, enquanto os estados contribuíam com os restantes $20 \%$. $^{2}$

O fortalecimento do processo de municipalização da saúde e a reforma fiscal efetivada pela Constituição Federal de $1988^{3}$ permitiram o crescimento da participação dos municípios no financiamento do SUS nos anos 90, em termos absolutos e relativos. Já os estados, nesta mesma década, além de reduzirem seu papel na oferta direta de ações e serviços de saúde, especialmente na atenção básica, tiveram parcela importante de suas receitas comprometidas com pagamento de dívidas, reduzindo sua participação dos gastos com saúde. Cabe destacar o papel importante da aprovação da Emenda Constitucional 29/2000, que ao vincular receitas para o setor saúde, permitiu a ampliação dos recursos alocados e maior responsabilização com o financiamento do SUS. ${ }^{4}$ Apesar disso, sua não regulamentação até o momento determinou uma redução do impacto sobre a ampliação efetiva do financiamento.

Cabe destacar que foi fundamental para a indução da ampliação da atenção básica a criação do Piso de Atenção Básica (PAB) com repasses de recursos para atenção básica com base em critérios populacionais, suprimindo o pagamento por produção de serviços neste nível. Este processo foi aprimorado com a criação do PAB variável, o estabelecimento de repasses em função do número de equipes de Saúde da Família, saúde bucal e agentes comunitários de saúde em atividade nos municípios. ${ }^{5}$

Mesmo concordando que a implantação das Norma Operacional Básica (NOB) 93 e 96 tiveram resultados positivos no fortalecimento do processo de descentralização e de expansão e reorganização da atenção básica-especialmente na NOB 96, com a criação do $\mathrm{PAB},{ }^{6}$ é necessário lembrar que sua implantação foi adiada por dois anos, entrando em vigor efetivamente apenas a partir de 1998 e que o valor originalmente proposto para o PAB em $\mathrm{R} \$ 12,00$ per capita por ano foi reduzido para um mínimo de $\mathrm{R} \$ 10,00$, praticado para a grande maioria dos municípios brasileiros que recebiam valor inferior a este, ficando entre $R \$ 10,00$ e $\mathrm{R} \$ 18,00$ para aqueles que apresentavam uma série histórica de produção de procedimentos de atenção básica acima do patamar mínimo estabelecido. ${ }^{5}$

Outro aspecto importante é que a implantação do PAB se deu após um período de quatro anos de gastos decrescentes do governo federal em programas de saúde, com estas despesas orçamentárias reduzindo em $21 \%$ entre 1995 e $1998 .^{7}$

Num primeiro momento a introdução do PAB produziu uma elevação dos recursos federais transferidos para a atenção básica para a maioria absoluta dos municípios brasileiros. Contudo, a defasagem do valor nos anos posteriores a sua implantação, o baixo nível de participação da esfera estadual no financiamento e o fato de não terem sido implantadas medidas que diferenciassem as necessidades do financiamento levando em conta as desigualdades intermunicipais, fizeram com que progressivamente o caminho da busca da eqüidade no financiamento deste nível de atenção fosse se distanciando. Estudos sobre a avaliação das mudanças ocorridas na atenção básica e as demandas dos gestores municipais apontavam a importância de que fossem adotadas medidas não apenas para aumentar o volume de recursos transferidos aos municípios, como também com vistas a permitir uma alocação eqüitativa de recursos, contemplando a necessidade de discriminar positivamente municípios com maiores necessidades de aporte para viabilizar a oferta de atenção à saúde em seu território. ${ }^{8}$

A busca da eqüidade tem sido um desafio permanente para a gestão de sistemas de saúde em diversos países. No caso brasileiro a própria Lei Orgânica da Saúde (Lei 8.080/90) já trazia, em seu artigo 35, critérios em relação ao financiamento do SUS contemplando as diferenças nos perfil epidemiológico e demográfico, na rede de saúde local, e por desempenho da gestão, entre outros. ${ }^{9}$

Entre as raras experiências de mudanças nos critérios de financiamento destaca-se a "Municipalização Solidária da Saúde" adotada pelo governo do Rio Grande do Sul, entre 1999 a 2002, que implementou repasses automáticos e regulares para fundos municipais de saúde com base em um conjunto de critérios envolvendo: tamanho da população, perfil demográfico, epidemiológico e características da rede de serviços. ${ }^{10}$

Ao final do ano de 2002 evidenciava-se intenso 
desfinanciamento da atenção básica, com o valor do Piso de Atenção Básica sem reajuste desde sua implantação, em 1998, nem a correção da base populacional para o cálculo em 2001 e 2002. Da mesma forma, os incentivos para Saúde da Família apresentavam os mesmos valores desde que haviam sido criados. $^{11}$

Em torno de $70 \%$ dos recursos para o custeio das equipes de Saúde da Família eram bancadas a partir de fonte municipal, com sério comprometimento da possibilidade de expansão e da qualidade das ações desenvolvidas frente das dificuldades dos municípios em ampliar ainda mais sua participação no financiamento, além dos constrangimentos impostos pela Lei de Responsabilidade Fiscal. Cabe ressaltar ainda, que os municípios brasileiros apresentam grande desigualdade na capacidade de geração de receitas próprias e o custeio, especialmente com pessoal de nível superior para atuar nas equipes de saúde da família, é mais elevado nos menores municípios, naqueles com piores indicadores de saúde, menor capacidade instalada de serviços de saúde e os localizados no interior e localidades de mais difícil acesso. Nesse cenário as transferências federais podem cumprir papel destacado na busca de eqüidade no âmbito do SUS, particularmente no tocante a organização das ações e serviços de atenção básica. $^{12}$

A partir de 2003, o Ministério da Saúde estabeleceu uma estratégia de correção progressiva, aumentando o PAB fixo, com seu valor mínimo saindo de $R$ \$ 10,00 para $R$ \$ 13,00, com metas de $R$ \$ 15,00 para o $2^{\circ}$ semestre de 2005 e de $\mathrm{R} \$ 18,00$ para o segundo semestre de 2006. O aumento para $\mathrm{R} \$$ 15,00 foi operacionalizado em setembro de 2006 (com um ano de atraso em relação ao previsto no Plano Plurianual do Ministério da Saúde). A partir de 2003, a correção da base populacional para cálculo do PAB fixo passou a ser feita anualmente. ${ }^{11}$

Além disso, em 2004 foi aprovada e implantada a incorporação nesta base de cálculo da população assentada entre os anos 2000 e 2003, após o Censo do Instituto Brasileiro de Geografia e Estatística (IBGE) em 2000. ${ }^{13}$

Os incentivos para Saúde da Família sofreram reajuste linear de 20\%, em 2003. Para os Agentes Comunitários de Saúde (ACS) foi criado um $13^{\circ}$ repasse. Além disso, foi alterada a tabela de incentivo de saúde da família para os municípios com mais de 100.000 habitantes. $^{11}$

Com objetivo de atingir um financiamento mais eqüitativo a Portaria do Ministério da Saúde (GM/MS no 1.434), de 14/07/04, estabeleceu um adicional de $50 \%$ no valor dos incentivos de Saúde da Família e Saúde Bucal para municípios com menos de 30.000 habitantes (menos de 50.000 na Amazônia Legal) e com Índice de Desenvolvimento Humano (IDH) menor ou igual a 0,7. Também foi implementado este diferencial no valor para as equipes que atuam em áreas de assentamentos rurais e de quilombolas em municípios que não se enquadravam nos critérios anteriores. Importante destacar que pela primeira vez foram empregados indicadores de condições sociais como critério para diferenciar os repasses federais aos municípios para financiamento do SUS. ${ }^{14}$

Tais medidas beneficiaram 3008 municípios $(54,06 \%)$, sendo que os 2281 municípios mais pobres do país passaram a ter $50 \%$ a mais nos valores dos incentivos para todas as suas equipes (41,01\% do total de municípios brasileiros) e 727 municípios para aquelas equipes que cobrem populações de assentamentos rurais e remanescentes de quilombos. ${ }^{15}$ (Tabela 1 e Figura 1 ).

Estas mudanças representaram um aumento médio de $35,93 \%$ no repasse para cada equipe de saúde da família (incluindo PAB fixo e incentivos para Saúde da Família, Agentes Comunitários de Saúde e Saúde Bucal). Este aumento chegou a $75 \%$ nos municípios que recebem o adicional de $50 \%$. $^{15}$ (Tabela 2).

Seguindo a mesma lógica, foram mudados os parâmetros de cobertura por ACS na zona rural de municípios da Amazônia Legal, onde a densidade demográfica é baixa, reduzindo o número de pessoas acompanhadas por cada ACS nestas áreas, com impacto potencial de ampliação de 7435 novos agentes comunitários de saúde. ${ }^{14}$

Foram também alterados os parâmetros de incentivos para saúde bucal, terminando com o limite de financiamento federal de uma equipe de saúde bucal para duas de saúde da família, com vistas a impulsionar de forma mais acelerada a expansão da atenção à saúde bucal através da estratégia de saúde da família. ${ }^{11}$

Os dados apresentados a seguir na homepage do DATASUS/Ministério da Saúde (www.datasus.gov.br) e tabulados empregando as ferramentas TABNET e TABWIN, com acessos realizados em setembro de 2006.

$\mathrm{O}$ valor médio anual repassado para cada equipe de saúde da família aumentou de $\mathrm{R}$ \$ 46.540,00 em 2002 para $\mathrm{R} \$ 66.883,00$ no ano de 2005 (aumento de $43,71 \%)$. Para cada equipe de saúde bucal cresceu de $\mathrm{R} \$ 13.275,00$, em 2002, para $\mathrm{R} \$$ $22.795,00$ em 2005 (71,71\% a mais). O valor per capita repassado pelo governo federal com vistas a sua participação no financiamento da atenção básica 
Tabela 1

Proporção de municípios beneficiados com 50\% a mais nos incentivos para equipes de saúde da família e de saúde bucal.

\begin{tabular}{|c|c|}
\hline Estado & $\begin{array}{c}\text { Proporção (\%) de } \\
\text { municípios beneficiados }\end{array}$ \\
\hline Paraíba & 93,72 \\
\hline Piauí & 93,69 \\
\hline Maranhão & 91,24 \\
\hline Amazonas & 90,32 \\
\hline Sergipe & 88,00 \\
\hline Acre & 86,36 \\
\hline Rio Grande do Norte & 85,03 \\
\hline Alagoas & 82,35 \\
\hline Amapá & 81,25 \\
\hline Tocantins & 79,14 \\
\hline Bahia & 78,66 \\
\hline Ceará & 68,48 \\
\hline Pará & 67,83 \\
\hline Pernambuco & 67,74 \\
\hline Roraima & 66,67 \\
\hline Rondônia & 53,85 \\
\hline Minas Gerais & 35,05 \\
\hline Espírito Santo & 23,08 \\
\hline Mato Grosso & 15,83 \\
\hline Paraná & 15,04 \\
\hline Goiás & 14,63 \\
\hline Mato Grosso do Sul & 9,09 \\
\hline Santa Catarina & 1,71 \\
\hline São Paulo & 1,40 \\
\hline Rio de Janeiro & 1,09 \\
\hline Rio Grande do Sul & 0,80 \\
\hline
\end{tabular}

Fonte: Brasil. Ministério da Saúde. Secretaria de Atenção à Saúde. Homepage do Banco de Dados do Sistema Único de Saúde-DATASUS. Disponível em: www.datasus.gov.br. [2006 set. 10]. ${ }^{15}$ foi aumentado em $44,11 \%$, passando de $\mathrm{R} \$ 22,85$ (2002) para $\mathrm{R} \$ 32,93$ em $2005^{15}$ (Tabela 3).

Em 2002 o orçamento para incentivos de Saúde da Família (incluindo agentes comunitários de saúde e equipes de saúde bucal) foi de 1,35 bilhões, tendo sido executado $93,85 \%$, chamando atenção o fato de que entre 2000 e 2002 a execução orçamentária dos recursos por parte do Ministério da Saúde para a estratégia de Saúde da Família foi abaixo do previsto em todos os anos, com apenas $92,16 \%$ de execução em 2001. Em 2005 este orçamento passou para aproximadamente 2,35 bilhões, tendo sido executado quase 2,5 bilhões, praticamente 100 milhões de reais a mais que a previsão $(106,14 \%)$. Entre 2002 e 2005 o orçamento para Saúde da Família aumentou em $74,06 \%$ e o incremento dos repasses federais para esta estratégia foi igual a $96,9 \%$ e a execução superando o orçamento previsto em todos os três anos. ${ }^{15}$ (Tabela 4).

O valor transferido a título de incentivo para equipes de saúde da família mais do que dobrou entre 2002 e 2005, saindo de R\$ 777.126.090,00 para $\mathrm{R} \$ 1.642 .923 .855,50$ (2,11 vezes maior). Para equipes de saúde bucal o aumento foi de 5,08 vezes, passando de R\$56.565.288,60 em 2002 para $\mathrm{R} \$ 287.288 .400,00$ em 2005. O total de recursos executado pelo Ministério da Saúde neste período foi ampliado em 52\%, aumentando de R\$ 3.990.708.286,82 em 2002 para R\$ 6.065.924.721,96 em 2005. É importante destacar que neste último ano o orçamento do Ministério da Saúde para Atenção Básica era de $\mathrm{R} \$$ 5.395.858.888,00 tendo sido, portanto, executado $12,5 \%$ a mais do que a previsão orçamentária ${ }^{15}$ (Tabela 5)

No período analisado os valores repassados pelo Piso de Atenção Básico Fixo (critério populacional)

Tabela 2

Repasses federais por Equipe de Saúde da Família, incluindo Saúde Bucal (mensal). Brasil, dezembro de 2002 a dezembro de 2004.

\begin{tabular}{lcccc}
\hline & PAB fixo* & Incentivo ESF & Incentivo ESB & Total \\
\hline Dezembro de 2002 & $4.166,67$ & $4.500,00$ & 541,50 & $9.208,17$ \\
Dezembro de 2004* & $5.416,67$ & $5.400,00$ & $1.700,00$ & $12.516,67$ \\
Dezembro de 2004** & $5.416,67$ & $8.100,00$ & $2.550,00$ & $16.066,67$ \\
\hline
\end{tabular}

* PAB = Piso de Atenção Básica; PAB fixo calculado para uma população de 5.000 pessoas; ** Municípios com cobertura de $70 \%$ do Saúde da Família; IDH = índice de desenvolvimento econômico <0,7 e <30 mil habitantes; ( $<50.000$ na Amazonia legal) e equipes que cobrem assentamentos rurais e quilombolas; equipe de saúde bucal sem Técnico de Higiene Dentária (THD); até 2002: máximo de uma Equipe de Saúde Bucal (ESB) para duas Equipes de Saúde da Família (ESF) - empregado 50\% do valor do incentivo (R\$1.083,00).

Fonte: Brasil. Ministério da Saúde. Secretaria de Atenção à Saúde. Homepage do Banco de Dados do Sistema Único de Saúde-DATASUS. Disponível em: www.datasus.gov.br. [2006 set. 10]. ${ }^{15}$ 
Proporção de municípios beneficiados com $50 \%$ a mais nos incentivos para Equipes de Saúde da Família e de Saúde Bucal, a partir da Portaria n. 1.434/GM de julho de 2004, por região.

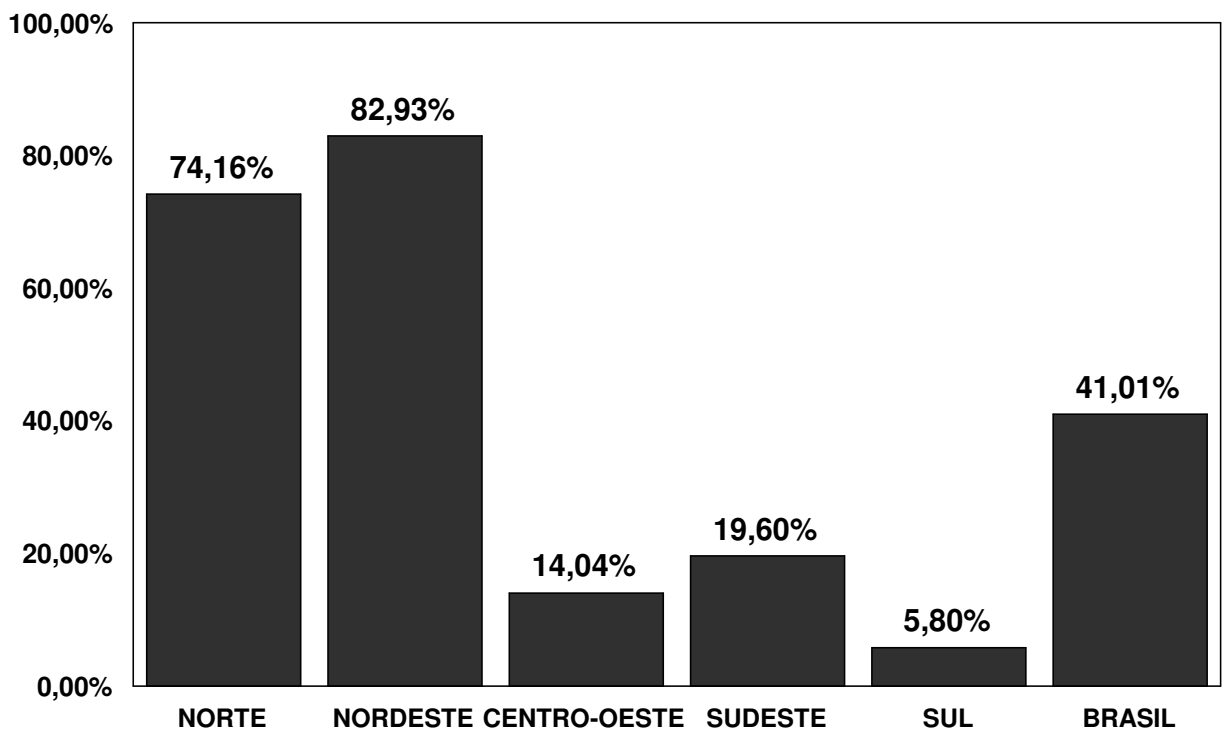

Fonte: Brasil. Ministério da Saúde. Portaria Nº 1.434/GM de 14 de julho de 2004. Define mudanças no financiamento da atenção básica em saúde no âmbito da estratégia Saúde da Família, e dá outras providências. Diário Oficial da União (DOU); 2004. ${ }^{14}$

Tabela 3

Valor médio anual repassado pelo governo federal por Equipe de Saúde da Família, por Equipe de Saúde Bucal e per capita. Brasil, 2002 a 2005.

\begin{tabular}{ccccccc}
\hline \multirow{2}{*}{ Ano } & \multicolumn{2}{c}{ Por Equipe Saúde da Família * } & \multicolumn{2}{c}{ Por Equipe Saúde Bucal ** } & \multicolumn{2}{c}{ Per capita Atenção Básica ** } \\
\cline { 2 - 7 } & $\begin{array}{c}\text { Valor médio } \\
(\mathrm{R} \$)\end{array}$ & $\begin{array}{c}\text { Incremento } \\
\text { anual }(\%)\end{array}$ & $\begin{array}{c}\text { Valor médio } \\
(\mathrm{R} \$)\end{array}$ & $\begin{array}{c}\text { Incremento } \\
\text { anual }(\%)\end{array}$ & $\begin{array}{c}\text { Valor médio } \\
(\mathrm{R} \$)\end{array}$ & $\begin{array}{c}\text { Incremento } \\
\text { anual }\end{array}$ \\
\hline 2002 & $46.540,00$ & & $13.275,00$ & & 22,85 & \\
2003 & $54.622,00$ & 17,37 & $14.735,00$ & 11,00 & 25,56 & 11,86 \\
2004 & $64.126,00$ & 17,40 & $21.032,00$ & 42,73 & 29,81 & 16,63 \\
2005 & $66.883,00$ & 4,30 & $22.795,00$ & 8,38 & 32,93 & 10,47 \\
\hline
\end{tabular}

* Equipe Saúde da Família: calculado com base no total anual de recursos repassados através do incentivo para equipes de saúde da família e do número de equipes ao final de cada ano; ** Equipe Saúde Bucal: calculado com base no total anual de recursos repassados através do incentivo para equipes de saúde bucal e do número de equipes ao final de cada ano; *** Per capita Atenção Básica: calculado com base no total anual de recursos repassados para atenção básica à saúde e na população estimada pelo Instituto Brasileiro de Geografia e Estatística para cada ano analisado.

Fonte: Brasil. Ministério da Saúde. Secretaria de Atenção à Saúde. Homepage do Banco de Dados do Sistema Único de Saúde-DATASUS. Disponível em: www.datasus.gov.br. [2006 set. 10]. ${ }^{15}$ 
Tabela 4

Evolução dos recursos financeiros federais (orçamento e execução financeira) para Estratégia de Saúde da Família. Brasil, 2000 a 2005.

\begin{tabular}{lccc}
\hline & Orçado & \multicolumn{2}{c}{ Executado } \\
\cline { 2 - 4 } Ano & Valor (R\$) & Valor (R\$) & $\%$ \\
\hline 2000 & $680.000 .000,00$ & $655.043 .329,14$ & 96,33 \\
2001 & $970.000 .000,00$ & $893.938 .793,64$ & 92,16 \\
2002 & $1.350 .000 .000,00$ & $1.266 .918 .379,80$ & 93,85 \\
2003 & $1.680 .000 .000,00$ & $1.686 .837 .003,28$ & 100,41 \\
2004 & $2.071 .000 .000,00$ & $2.185 .425 .264,50$ & 105,53 \\
2005 & $2.349 .794 .994,00$ & $2.494 .120 .465,43$ & 106,14 \\
\hline
\end{tabular}

Inclui incentivos para Agentes Comunitários de Saúde, Equipes de Saúde da Família e Equipes de Saúde bucal; repasses para saúde da família tiveram aumento entre 2002 e 2005 igual a $+97 \%$

Fonte: Brasil. Ministério da Saúde. Secretaria de Atenção à Saúde. Homepage do Banco de Dados do Sistema Único de Saúde-DATASUS. Disponível em: www.datasus.gov.br. [2006 set. 10]. ${ }^{15}$

Tabela 5

Valor total anual repassado pelo governo federal através do incentivo para Equipes de Saúde da Família, Equipes de Saúde Bucal e total repassado para atenção básica. Brasil, 2002 a 2005.

\begin{tabular}{lccccc}
\hline \multirow{2}{*}{ Ano } & Incentivo Equipe Saúde da Família* & Incentivo Equipe Saúde Bucal ** & Total Atenção Básica ** \\
\cline { 2 - 6 } & Valor (R\$) & $\begin{array}{c}\text { Incremento } \\
\text { anual (\%) }\end{array}$ & Valor (R\$) & $\begin{array}{c}\text { Incremento } \\
\text { anual (\%) }\end{array}$ & $\begin{array}{c}\text { Valor (R\$) } \\
\text { Incremento } \\
\text { anual (\%) }\end{array}$ \\
\hline 2002 & $777.126 .090,00$ & & $56.565 .288,60$ & & $3.990 .708 .286,82$ \\
2003 & $1.041 .528 .472,00$ & 34,02 & $90.915 .931,28$ & 60,73 & $4.520 .355 .354,27$ \\
2004 & $1.361 .520 .555,00$ & 30,72 & $188.257 .650,00$ & 107,07 & $5.339 .202 .917,63$ \\
2005 & $1.642 .923 .855,50$ & 20,67 & $287.288 .400,00$ & 52,60 & $6.065 .924 .721,96$ \\
\hline
\end{tabular}

* Equipe Saúde da Família: calculado com base no total anual de recursos repassados através do incentivo para equipes de saúde da família e do número de equipes ao final de cada ano; ** Equipe Saúde Bucal: calculado com base no total anual de recursos repassados através do incentivo para Equipes de Saúde Bucal e do número de equipes ao final de cada ano; *** Total Atenção Básica: calculado com base no total anual de recursos repassados para atenção básica à saúde e na população estimada pelo Instituto Brasileiro de Geografia e Estatística (IBGE) para cada ano analisado.

Fonte: Brasil. Ministério da Saúde. Secretaria de Atenção à Saúde. Homepage do Banco de Dados do Sistema Único de Saúde-DATASUS. Disponível em: www.datasus.gov.br. [2006 set. 10]. ${ }^{15}$

aumentaram em $32,17 \%$-de cerca de 1,766 bilhões de reais para 2,335 bilhões em 2005. Já os incentivos (PAB variável) tiveram incremento de $110,88 \%$, saindo de 1,27 bilhões de reais para aproximadamente 2,7 bilhões em 2005. Os demais repasses para atenção básica cresceram em menor proporção $(10,32 \% \text { neste período })^{15}$ (Tabela 6$)$.

Comparando recursos federais para atenção básica com os da atenção especializada ambulatorial e hospitalar (MAC)-sem o Fundo de Ações Estratégicas (FAEC)-observa-se que os da atenção básica representaram $18,6 \%$ do total gasto com MAC em 1998, 27,1\% em 1999, ficando em 32,6\% e $35,6 \%$ entre 2000 e 2002. Em 2003 cresceu para $38,8 \%$, em 2004 para 44\%, e em 2005 foi igual a $47,2 \%$, chegando a um montante próximo a $50 \%$ 
Repasses financeiros federais para atenção básica no Sistema Único de Saúde: Piso de Atenção Básica (PAB) variável, PAB fixo e outros repasses. Brasil, 2002 a 2005.

\begin{tabular}{lccccccccc}
\hline Ano & \multicolumn{2}{c}{ Piso de Atenção Básica variável } & \multicolumn{2}{c}{ Piso de Atenção de Básica fixo } & \multicolumn{2}{c}{ Outros } & Total Atenção Basica \\
\cline { 2 - 7 } & $\begin{array}{c}\text { Valor (x R\$ } \\
1.000 .000)\end{array}$ & $\begin{array}{c}\text { Incremento } \\
\text { anual (\%) }\end{array}$ & $\begin{array}{c}\% \text { do } \\
\text { Total }\end{array}$ & $\begin{array}{c}\text { Valor (x R\$ } \\
1.000 .000)\end{array}$ & $\begin{array}{c}\text { Incremento } \\
\text { anual (\%) }\end{array}$ & $\begin{array}{c}\% \text { do } \\
\text { Total }\end{array}$ & $\begin{array}{c}\text { Valor (x R\$ } \\
1.000 .000)\end{array}$ & $\begin{array}{c}\text { Incremento } \\
\text { anual (\%) }\end{array}$ \\
\hline 2002 & $1.270,5$ & & 31,84 & $1.766,8$ & & 44,27 & 953,408 & $3.990,708$ \\
2003 & $1.662,8$ & 30,88 & 36,78 & $1.902,9$ & 7,70 & 42,10 & 954,655 & 0,13 & $4.520,355$ \\
2004 & $2.191,0$ & 31,77 & 41,04 & $2.134,5$ & 12,17 & 39,98 & $1.013,7$ & 6,19 & $5.339,203$ \\
2005 & $2.679,2$ & 22,28 & 44,17 & $2.335,1$ & 9,40 & 38,50 & $1.051,82$ & 3,76 & $6.065,925$ \\
2006 & $3.248,5$ & 21,26 & & $2.470,3$ & 5,79 & & & & \\
\hline
\end{tabular}

Fonte: Brasil. Ministério da Saúde. Secretaria de Atenção à Saúde. Homepage do Banco de Dados do Sistema Único de Saúde-DATASUS. Disponível em: www.datasus.gov.br. [2006 set. 10]. ${ }^{15}$

dos valores aplicados em MAC. Incluindo-se recursos do FAEC aos do MAC, estes percentuais ficaram entre 32,6 e $33,8 \%$ de 2000 a 2002 , aumentando em 2003 para $35,1 \%$, em 2004 para $37,9 \%$, e para $40,2 \%$ em 2005, ou seja, um volume igual a mais de $40 \%$ de todos os repasses feitos pelo Ministério da Saúde para financiamento da atenção ambulatorial e hospitalar de média e alta complexidade $^{15}$ (Tabela 7)

Importante também identificar que a partir de 2003 ocorreu a implantação do Projeto de Expansão e Consolidação do Saúde da Família (PROESF), com objetivo de impulsionar a expansão e a qualificação desta estratégia, com um orçamento para oito anos de US\$ 550 milhões de dólares. Um dos seus componentes dirigido para os municípios com mais de 100 mil habitantes, que concentram cerca de $55 \%$ da população brasileira, financia a melhoria da infraestrutura das unidades de saúde da família e de serviços públicos de média complexidade ambulatorial, assim como o fortalecimento da capacidade de gestão municipal, a capacitação dos recursos humanos e a avaliação do Programa de Saúde da Família (PSF) como um requisito obrigatório. Outros dois componentes estão direcionados para o total dos municípios e equipes de saúde da família visando ações de educação permanente dos recursos humanos, ações de monitoramento e avaliação e fortalecimento da capacidade dos estados em prestarem cooperação técnica e acompanhamento das ações desenvolvidas nos municípios. ${ }^{11}$

Concluindo, cabe registrar que o aumento dos repasses federais para atenção básica, em especial para a estratégia de Saúde da Família, incluindo saúde bucal, possibilitou uma forte indução para
Tabela 7

Comparação entre recursos federais para atenção básica em relação aos recursos para média e alta complexidade (com e sem os recursos do Fundo de Ações Estratégicas (FAEC). Brasil, 1998 a 2005.

\begin{tabular}{lcc}
\hline Ano & $A B /$ MAC (\%) & $A B /(M A C+F A E C)(\%)$ \\
\hline 1998 & 18,60 & 18,60 \\
1999 & 27,10 & 27,10 \\
2000 & 32,60 & 32,60 \\
2001 & 33,90 & 33,50 \\
2002 & 35,60 & 33,80 \\
2003 & 38,80 & 35,10 \\
2004 & 44,00 & 37,90 \\
2005 & 47,20 & 40,20 \\
\hline
\end{tabular}

MAC (média e alta complexidade ambulatorial e hospitalar): transferências para estados e municípios e pagamentos por produção de serviços para alta e média complexidade (não inclui Ações Estratégicas-FAEC); MAC+FAEC: transferências para estados e municípios e pagamentos por produção de serviços para alta e média complexidade, incluindo Ações Estratégicas-FAEC.

Fonte: Brasil. Ministério da Saúde. Secretaria de Atenção à Saúde. Homepage do Banco de Dados do Sistema Único de Saúde - DATASUS. Extraído de [www.datasus.gov.br], acesso em [setembro de 2006]. 
ampliação do número de equipes em atividade. Entre dezembro de 2002 e junho de 2006 observa-se um aumento de 55,5\% no número de equipes de saúde da família, 221,7\% no número de equipes de saúde bucal e $22,6 \%$ no número de agentes comunitários de saúde. ${ }^{15}$

A análise dos dados aqui apresentados eviden-

\section{Referências}

1. Bodstein R. Atenção básica na agenda da saúde. Ciênc Saúde Coletiva. 2002; 7: 401-12.

2. Ugá MAD, Santos IS. Uma análise da progressividade do financiamento do Sistema Único de Saúde (SUS). Cad Saúde Pública. 2005; 22: 1597-609.

3. Brasil. Constituição de 1988. 17.ed.atualizada em 2001. Brasília, DF: Câmara dos Deputados; 2001.

4. Faveret ACSC. A vinculação constitucional de recursos para a saúde: avanços, entraves e perspectivas. Ciênc Saúde Coletiva. 2003; 8: 371-8.

5. Levcovitz E, Lima LD, Machado CV. Política de saúde nos anos 90: relações intergovernamentais e o papel das Normas Operacionais Básicas. Ciênc Saúde Coletiva. 2001; 6: 269-91.

6. Melamed C, Costa NR. Inovações no financiamento federal à Atenção Básica. Ciênc Saúde Coletiva. 2003; 8: 393-401.

7. Costa RCR. Descentralização, financiamento e regulação: a reforma do sistema público de saúde no Brasil durante a década de 1990. Rev Sociol Política. 2002; 18: 49-71.

8. Machado ENM, Fortes FBCTP, Somarriba M. Efeitos da introdução do $\mathrm{PAB}$ sobre a distribuição de recursos e a prestação de serviços: o caso de Minas Gerais. Ciênc Saúde Coletiva. 2004; 9: 99-111.

9. Brasil. Lei $\mathrm{n}^{\circ} 8.080$, de 19 de setembro de 1990. Dispõe sobre as condições para a promoção, proteção e recuperação da saúde, a organização e o funcionamento dos serviços correspondentes. Diário Oficial da União (DOU). 199020 set; Seção 1.

Recebido em 12 de junho de 2007

Versão final apresentada em 11 de setembro de 2007

Aprovado em 13 de outubro de 2007 ciou não apenas um aumento de cobertura pela estratégia de saúde da família no período analisado, como também uma maior participação da atenção básica no orçamento federal do SUS e a incorporação de medidas que apontam na direção da eqüidade com financiamento diferenciado para os municípios mais pobres do país.

10. Pelegrini MLM, Castro JD, Drachler ML. Eqüidade na alocação de recursos para a saúde: a experiência do Estado do Rio Grande do Sul, Brasil. Ciênc Saúde Coletiva. 2005; 10: 275-86.

11. Brasil. Ministério da Saúde. Balanço da Saúde. Brasília,DF; 2005.

12. Marques RM, Mendes A. Atenção Básica e Programa de Saúde da Família (PSF): novos rumos para a política de saúde e seu financiamento? Ciênc Saúde Coletiva. 2003; 8: 403-15.

13. Brasil. Ministério da Saúde. Portaria No $1.432 / G M$, de 14 de julho de 2004. Inclui, na base de cálculo do valor do Piso de Atenção Básica (PAB -fixo), a população assentada entre os anos 2000 e 2003, e dá outras providências. Diário Oficial da União (DOU). 200415 jul; Seção 1: 31-2.

14. Brasil. Ministério da Saúde. Portaria No 1.434/GM de 14 de julho de 2004. Define mudanças no financiamento da atenção básica em saúde no âmbito da estratégia Saúde da Família, e dá outras providências. Diário Oficial da União (DOU). 2004; Seção 1: 36.

15. Brasil. Ministério da Saúde. Secretaria de Atenção à Saúde. Homepage do Banco de Dados do Sistema Único de SaúdeDATASUS. Disponível em: www.datasus.gov.br. [2006 set. $10]$. 